\title{
UK contract staff need help, says report
}

[LONDON] Principal investigators in the United Kingdom are being urged to do more to ensure that contract researchers working on projects under their responsibility receive proper guidance on career prospects. This might include the steps necessary to move into a non-academic career.

Similarly, bodies such as the research councils, which support contract researchers, and the higher-education institutions at which most of them work, have been encouraged to increase their own efforts to ensure that such individuals are aware of the support already on offer.

For example, it is recommended that every higher-education institution should establish a clear focus for contract research staff. And research councils have been asked to consider whether grant evaluation procedures might be adopted to reward effective management and career guidance by the institutions.

These are among the proposals contained in a report published last week by the Research Careers Initiative (RCI), which was set up in 1996 to oversee the implementation of a 'concordat' between representatives of higher-education institutions and research councils on the career management of contract researchers.

The signing of the concordat reflected a growing recognition that the issue is a major problem for the research community. It is calculated that there are currently 30,000 researchers in British universities working on contracts of an average of three years, compared with 70,000 full-time staff.

In some disciplines the figures are even more dramatic; indeed, in science and engineering there are around two contract researchers for every three full-time acad-

\section{Main recommendations}

The main recommendations of the Research Careers

Initiative are that:

- A clear focus should be established on policy for contract research staff at every institution of higher education.

- Institutions should obtain feedback on questionnaires completed by contract researchers about their working practices. - A guide to best practice produced by the $\mathrm{RCI}$ should be widely publicized. - Careers guidance resources should

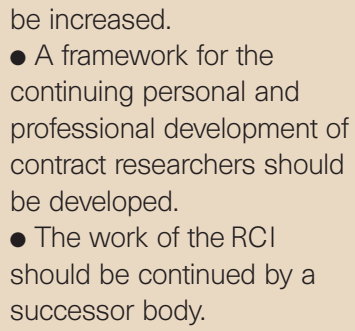

emic researchers. Many take on a succession of contract positions in the hope of eventually being appointed to an academic post; at present, only 20 per cent achieve this.

"It is very important to manage expectations," says David Clark of the Engineering and Physical Sciences Research Council (EPSRC). "Contract researchers should not expect that the next step is automatically an academic position."

The EPSRC has taken the lead in introducing measures to ensure that contract researchers are allowed to develop their careers in a way that will make them attractive to potential employers outside universities. For example, it has agreed to pay for contract researchers to go on certain career development courses.

Gareth Roberts, vice-chancellor of the University of Sheffield and chairman of the $\mathrm{RCI}$, says in an introduction to the report that such initiatives and other moves by universities themselves illustrate that "measurable progress" has been made since the concordat was signed. But he admits that "problems persist".

The report and the surveys on which it is based describe some of the problems. For

\section{New telescope shows off its paces}

[LONDON] This image of the Dumbbell Nebula (right) has been obtained by the European Southern Observatory's Very Large Telescope on Mount Paranal in Chile. The Dumbbell Nebula is located in the Vulpecula constellation, believed to be around 1,200 light years from Earth. It consists of very rarified gas that has been ejected from a hot central star, visible in the image, and which is now in one of the final stages of its evolution.

The nebula's gas atoms are heated by the intense ultraviolet radiation from the star, and emit radiation at specific wavelengths. Filters on the telescope allow the isolation of emissions from particular atoms and ions, shown in the intricate structure of the central part of the nebula.

The three-colour composite image was

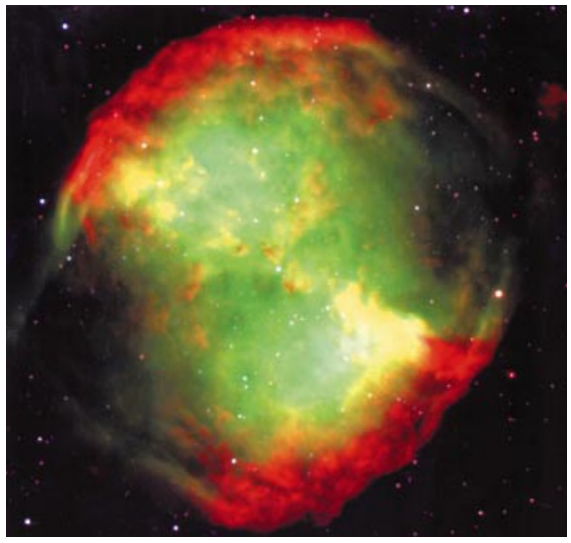

taken last month during the telescope's first commissioning phase, in which bright objects were recorded. The second phase will look at faint objects.
Ehsan Masood example, it says that although the number of higher-education institutions taking action is growing, the proportion involved is still "far too low". It suggests that research funders should adopt "a more pro-active role".

Questionnaires distributed among contract researchers revealed that they usually receive little career guidance. Although this is partly because there is little such guidance, the RCI admits that it is also the result of a disinclination among many to seek it.

An RCI working group identified several reasons for the low participation in career guidance activities. These range from a lack of support among research managers, to concern that an expression of interest in an alternative career could destroy already slim prospects of an academic career.

A separate working group on training argues that there seems to be a weakness in the research management chain at the level of principal investigator. According to the working group, principal investigators are often under great pressure to achieve a high level of research output and to meet contractual commitments to research sponsors. "In these circumstances, the personal and development needs of contract research staff are not always high on the agenda."

The RCI's main report has been welcomed by the Association of University Teachers, the labour union representing the interests of university staff - including contract researchers. "Its recommendations offer hope of real progress in careers guidance and professional development for university researchers," says the union's general secretary David Triesman.

Triesman goes further, however, arguing against what he calls "the contract culture", where basic insecurity creates "the inefficiency of high turnover, wastage, non-completion and inadequate dissemination of research".

Although, in publishing the report, the RCI has achieved the objective it was set up for, one recommendation is that it be reconstituted as a smaller group to help take forward some of the proposals in its report.

The full text of the report can be found on: www.cvcp.ac.uk

DavidDickson 